\title{
Self-poisoning of crystal nuclei in hard-rod liquids
}

\author{
Tanja Schilling and Daan Frenkel \\ FOM Institute for Atomic and Molecular Physics, Kruislaan 407, 1098 SJ Amsterdam, \\ The Netherlands
}

Received 3 December 2003

Published 30 April 2004

Online at stacks.iop.org/JPhysCM/16/S2029

DOI: $10.1088 / 0953-8984 / 16 / 19 / 014$

\begin{abstract}
We report on a Monte Carlo study of the pathway for crystal nucleation in a fluid of short, hard, colloidal rods. In the earliest stages of nucleation, a single-layered lamellar crystallite forms. Subsequent thickening of this lamella is hampered by the fact that the top and bottom surfaces of the crystallite are preferentially covered by rods that align parallel to the surface. As a single lamella is thermodynamically not stable, subsequent growth of individual crystals is stunted. Recently experimental evidence for such stunted crystal growth has been reported by Maeda and Maeda (2003 Phys. Rev. Lett. 90 018303) for experiments on suspensions of colloidal rods.
\end{abstract}

\section{Introduction}

Recently pathways and rates of crystal nucleation have been obtained by computer simulation for several systems of spherical colloidal particles [1]. When spherical particles crystallize, the crystal nuclei that form tend to be roughly spherical [1,2]. In contrast, non-spherical molecules (as for example many proteins or the building blocks of liquid crystals) may form crystals that are strongly anisometric. This has two reasons: first of all, the solid-liquid interfacial free-energy density may be very different for crystal faces parallel and perpendicular to the molecular axes. As a consequence, the equilibrium shape of crystal nuclei is non-spherical. In addition, once crystals grow beyond the size of the critical nucleus, the rate at which molecules are incorporated into the crystal may depend strongly on the nature of the crystal face. In some extreme cases, this may lead to the formation of extremely elongated (liquid) crystal fibres $[3,4]$.

In some crystallization processes the growth rate of a crystal facet can be inhibited by the addition of an impurity that strongly adsorbs onto the growth front and thereby 'poisons' the incorporation of new molecules into that facet. In fact, the molecules that lead to poisoning of a growth surface need not be impurities in the chemical sense. They can simply be misaligned or misfolded specimens of the crystallizing species (see e.g. [5, 6]). In either case, one can argue that the crystal growth is blocked by incorporation of defects in the crystal. In this paper, we report a numerical study that reveals a different kind of self-poisoning: the crystal nucleus itself is free of defects, but it is covered by a liquid layer of molecules that do not have the 


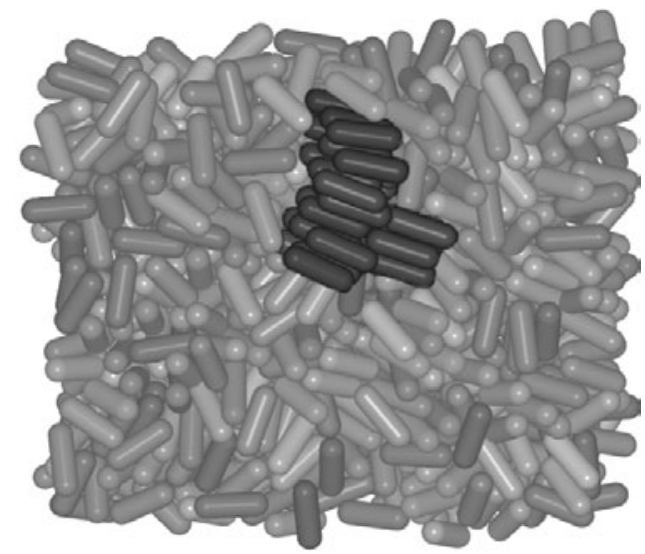

Figure 1. A metastable liquid configuration at $p=6$, containing a (subcritical) crystalline cluster (dark).

correct orientation to be incorporated into the crystal. This has consequences both for crystal nucleation and for subsequent crystal growth.

\section{Model and simulation technique}

The system that we studied is a model for a colloidal suspension of hard, rod-like particles. These particles are modelled as hard sphero-cylinders with a diameter $D$ and a cylindrical segment of length $L$. In our simulations, we considered 2400 rods with an aspect ratio $L / D=2$. Short, hard sphero-cylinders have a relatively simple phase diagram [7]: at low pressures, the system forms an isotropic fluid, at high pressures it forms an orientationally ordered crystal. The transition between the two phases is of first order. At a pressure $p=5.64 k_{\mathrm{B}} T / D^{3}$, the isotropic fluid coexists with the crystal phase. (In the following the unit of energy is $k_{\mathrm{B}} T$ and the unit of length is $D$.)

We are interested in the nucleation of a crystal from the metastable fluid phase. To study this phenomenon, we compressed the fluid beyond coexistence, to a pressure of $p=6$. At this pressure, the chemical potential difference between the (metastable) fluid and the solid phases is approximately $0.2 k_{\mathrm{B}} T$ /particle. In spite of the fact that, at this pressure, the solid is more stable than the liquid, spontaneous crystallization never occurred on the timescales of our (quite long) simulations. However, at higher supersaturations (e.g. $p=8, \Delta \mu=1.3 k_{\mathrm{B}} T /$ particle) spontaneous crystallization did occur on the timescale of a simulation. By working at $p=6$, a pressure where spontaneous crystallization is suppressed, we can study the free energy and the structure of small crystal nuclei (see figure 1), using the biased sampling techniques described in $[1,2]$.

The basic idea behind these techniques is the following: one defines a local orderparameter, which distinguishes the liquid phase from all possible solid phases which might occur along the nucleation path. With this parameter, clusters of 'solid-like' particles are identified. Then the simulation is biased such that it produces on average a given size for the largest solid-like cluster in the system. For a series of biased simulations, histograms of cluster size and structure are measured and combined into the complete nucleation barrier.

In order to follow the formation of crystallites from solution, we need an order-parameter that distinguishes particles in a crystalline environment from those in the liquid phase. In the 


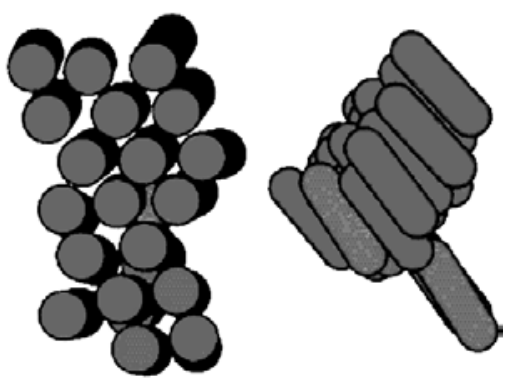

Figure 2. Top and side view of a typical crystalline cluster. Most rods are incorporated in a single crystalline layer.

crystal phase, all particles within one crystal tend to be strongly aligned. We employed this fact to define our crystallinity criterion: two particles $i$ and $j$ are said to belong to the same crystallite if (a) their surface-to-surface distance is less than $0.5 \mathrm{D}$ and (b) the dot product of the unit vectors along their respective axes satisfies $\left|\mathbf{u}_{i} \cdot \mathbf{u}_{j}\right|>0.995$. (The choice of the exact value of this threshold is arbitrary, as long as it distinguishes between the solid and the fluid phase. We found our results to be stable against variation of this value.) With this criterion, $99.8 \%$ of all particles in an equilibrium crystal are recognized as belonging to that crystal. As the criterion only considers the relative orientation of neighbouring particles, it does not distinguish between crystalline, smectic and (dense) nematic structures. This means that we do not impose strong constraints on the structure of the crystal nucleus, but let the system find its own path.

In the liquid, many particles are identified as belonging to small crystallites. However, we found that the number of rods in these crystallites was rarely larger than 2: about $5 \%$ of all particles belong to 'crystal' dimers and less than one in a thousand belongs to a larger cluster. We never observed spontaneous fluctuations that resulted in crystalline clusters containing more than 15 particles.

We denote the average number of clusters consisting of $n$ particles by $N_{n}$. The probability of finding a cluster of size $n$ per unit volume is equal to $(N / V)\left(N_{n} / N\right)$, where $N$ is the total number of particles in the system. We measure $P(n) \equiv N_{n} / N$ and define an intensive freeenergy barrier for the formation of a crystal nucleus of $n$ particles as $\Delta G(n)=-k_{\mathrm{B}} T \ln P(n)$. To probe $P(n)$ for $n>15$, we use biased, multi-histogram simulations (for details, see [1]).

\section{Results}

When we perform such a multi-histogram simulation, we find that we can grow large, ordered clusters of up to 80 particles. However, somewhat surprisingly, we find that most particles in the ordered cluster are located in a single hexagonally ordered layer (see e.g. figure 2). As we bias the cluster-size distribution towards larger crystallites, we observe no tendency to form crystallites that contain multiple layers. To be more precise: a few particles may order on top of the crystallite, but these embryonic new layers quickly dissolve again. All growth of the cluster concentrates on the edges, and the free energy of the cluster rises monotonically with size (see figure 3). In other words: the system never crosses a nucleation barrier beyond which crystallites would grow spontaneously.

Interestingly, a rough analysis of the data shown in figure 3 suggests that a flat crystallite is not the most stable one. If we assume that the crystallite is cylindrical, with a thickness 


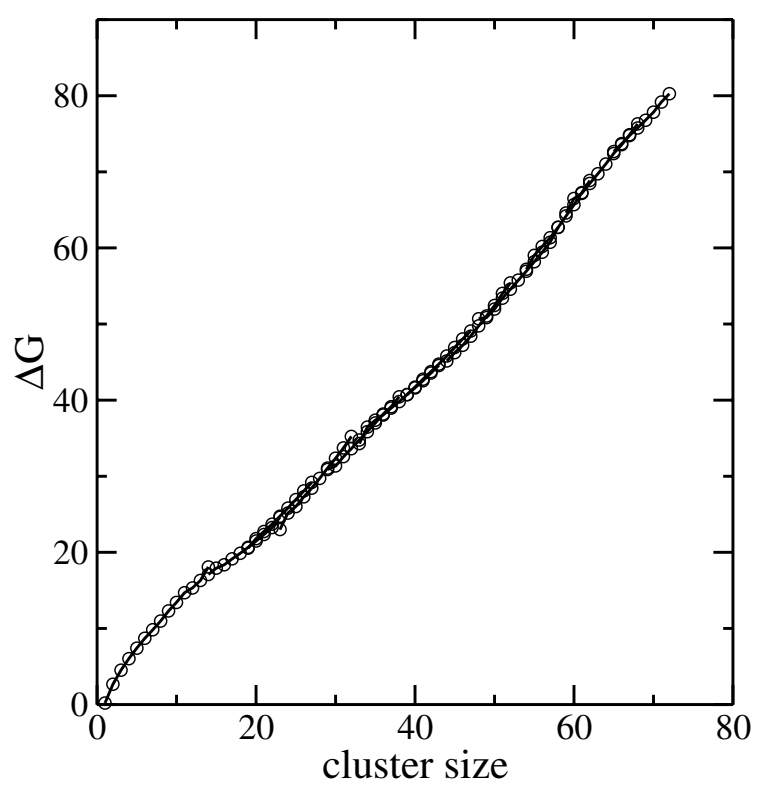

Figure 3. Size dependence of the free energy of a crystal nucleus. For the low supersaturations that we studied, $\Delta G$ for a single crystalline layer grows monotonically with cluster size. In order to nucleate a stable crystal, a multilayer nucleus must first form.

$h \equiv L+D$ and a radius $r$, then we can approximate the free energy of this cluster by:

$$
\Delta G(r)=2 \pi r h \gamma_{\|}+\pi r^{2}\left(2 \gamma_{\perp}-h \rho_{\mathrm{s}}|\Delta \mu|\right)
$$

where $\gamma_{\|}$and $\gamma_{\perp}$ are, respectively, the free-energy densities of the side and of the top/bottom surfaces. $\rho_{\mathrm{S}}$ is the density of the crystalline phase.

In a lamellar crystal, both the bulk free energy and the (dominant) surface free-energy scale with the cross-sectional area. A lamella can only grow spontaneously when $\left(2 \gamma_{\perp}-\rho_{\mathrm{s}}|\Delta \mu|\right)<$ 0 . At the low supersaturation that we use $(|\Delta \mu|=0.2)$, this condition is not satisfied. In fact, a fit of equation (1) to the free-energy barrier in figure 3 yields $\gamma_{\perp}=0.6$. Moreover, we find that $\gamma_{\|} \ll \gamma_{\perp}$. This is interesting, because in this case, we should expect multilayer clusters to be more stable than flat discs. The fact that, in our simulations, we observe the formation of disc-like crystallites suggests that there are kinetic reasons that inhibit the formation of multilayer crystallites. The simplest assumption is that multilayer growth is difficult because every new layer has to nucleate on top of an existing layer [4]. To test this, we used the same biased Monte Carlo procedure to compute the free energy of a crystallite that forms on top of an ordered crystalline substrate. However, before we discuss these simulations, it is instructive first to look at the ordering in the liquid near the crystal surface.

Figure 4 shows the angular distribution of particles in the liquid close to the top surface (the surface perpendicular to the director field) and the side surface (the surface parallel to it) of a perfect crystal of hard sphero-cylinders. Both distributions peak for $|\cos \theta|=1$, where particles are aligned with the director. However, for the top surface there is also a broad peak at $\cos \theta=0$, which indicates that a large fraction of the particles align parallel to the surface.

The preference of particles to lie flat on the crystal surface facilitates growth of the side surface, but it makes growth of the top/bottom surface difficult. Particles have to overcome a barrier of roughly $1.5 \mathrm{kT} /$ particle in order to stand up on the surface and align with the director. In contrast, incorporation of particles on the side face of a crystal is relatively easy. 


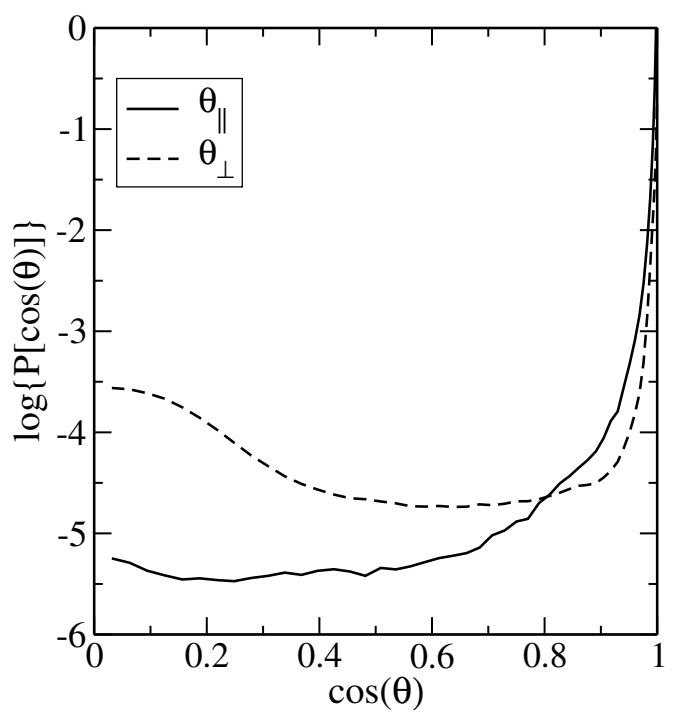

Figure 4. Angular distributions of liquid particles close to a surface of a hard-rod crystal. $\theta_{\|}$ denotes the surface parallel to the director field ('side surface'), $\theta_{\perp}$ the surface perpendicular to it ('top surface').

Indeed, we find no inhibition of the lateral growth of sphero-cylinder crystals, even when the growth of additional layers is effectively suppressed.

Recent work by Downton and Allen indicates that substrate roughness has a marked effect on the angular distribution functions of 'near-surface' particles [8]. In our work, we have not been able to investigate the dependence of surface roughness on supersaturation, because the simulations were done in a non-equilibrium situation. However, it would be very interesting to investigate how supersaturation influences nucleation behaviour via the roughness of the lamellae.

\section{Additional biases}

The discussion above suggests that, in order to form a second crystal layer, an appreciable fraction of the particles on the top or bottom surface must spontaneously align parallel to the surface normal. The 'reaction' path for second-layer formation would then be orientational alignment, followed by crystallization. To explore this possible route, we performed simulations where we introduced a second biasing parameter that facilitates the alignment of the particle orientation $\vec{u}_{i}$ with the surface normal $\vec{n}$, for particles close to the (top or bottom) surface.

$$
S_{\text {liquid }}=\left\langle(\vec{n} \cdot \vec{u})^{2}\right\rangle .
$$

Unlike the order-parameter that we used to measure crystallinity, $S$ increases continuously with alignment. This implies that $S$ is sensitive to the pre-alignment that is, presumably, a prerequisite for crystallization.

Indeed, we find that applying an orientational bias greatly facilitates the growth of a second crystal layer.

There is, however, another effect that slows down the growth of a second layer. Figure 5 shows the free-energy barrier associated with the formation of a compact crystalline island on 


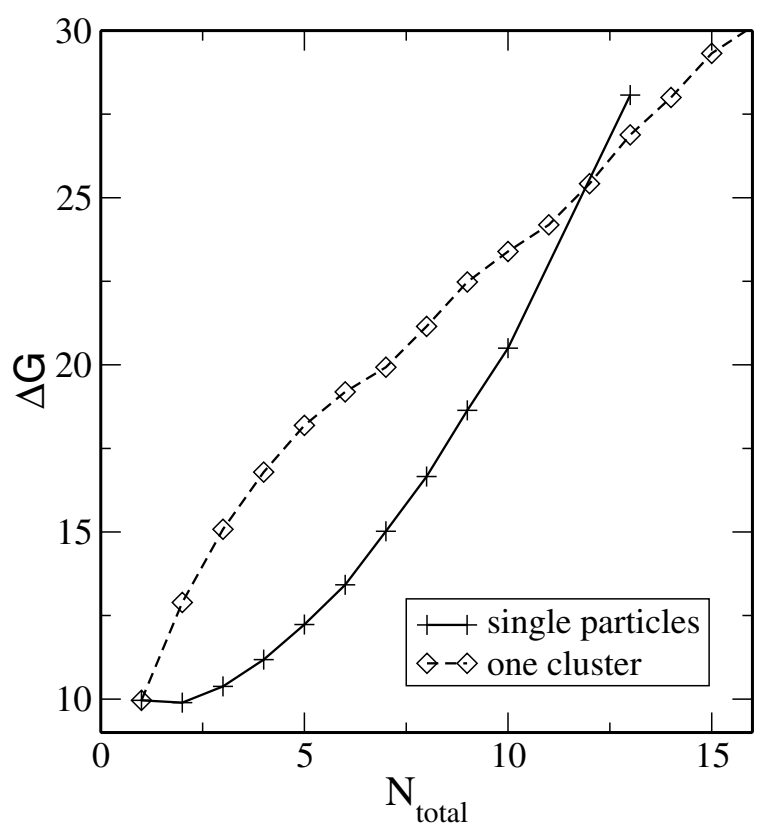

Figure 5. Free-energy dependence on particle distribution in the new layer: all particles in the new layer form one single cluster (dashed curve) and all particles are in clusters of size 1 (solid curve).

top of a lamellar substrate. In the same figure, we also show the free-energy cost associated with the addition of the same number of crystalline (i.e. well-aligned) particles, $N_{\text {total }}$, anywhere in the second layer. Clearly, the random distribution of single crystalline particles in the second layer has a higher entropy than the arrangement in a single cluster. This means that if a small number of crystalline particles is added to a second layer, it is entropically favourable to distribute them randomly over the surface. Once their number exceeds a certain threshold (12 for the case plotted in figure 5), surface tension wins over positional entropy and they aggregate into a cluster. This aggregation is, itself, a nucleation event.

Hence, second-layer growth is difficult for two reasons: it costs free energy to pre-align the particles that are to be incorporated in the crystal and then a nucleation event is needed to create a compact cluster that can grow to a larger size. We do not show the complete nucleation barrier for a second layer as, for the small supersaturation used in our simulations, the critical cluster size is rather large, $N_{\text {cluster }} \simeq 200$. (This high number is due to the strong line tension of the contact line between the cluster and the substrate. The sharp corner formed there restricts the possible orientations for particles in the liquid strongly.) By introducing a bias on $N_{\text {cluster }}$ it is possible to grow a complete second layer on top of the first one. Figure 6 shows a configuration snapshot of those particles which are close to the first layer (looking down onto the layer through the invisible liquid). Most particles lie flat on the first layer (light shades of grey), but there is also a cluster of roughly 50 standing particles (dark).

We do not, however, assume that the true pathway for crystal formation in experiments proceeds via the formation of additional layers on top of the first aggregate. As we have shown in this work, this would be a very unlikely path. Instead crystallization probably proceeds via the combination of several disc-like crystallites into a multilayered crystallite.

Recent experiments by Maeda and Maeda [9] on the isotropic-to-smectic transition in colloidal hard rods of $\beta$-FeOOH rods, showed that these particles tend to form disc-like 


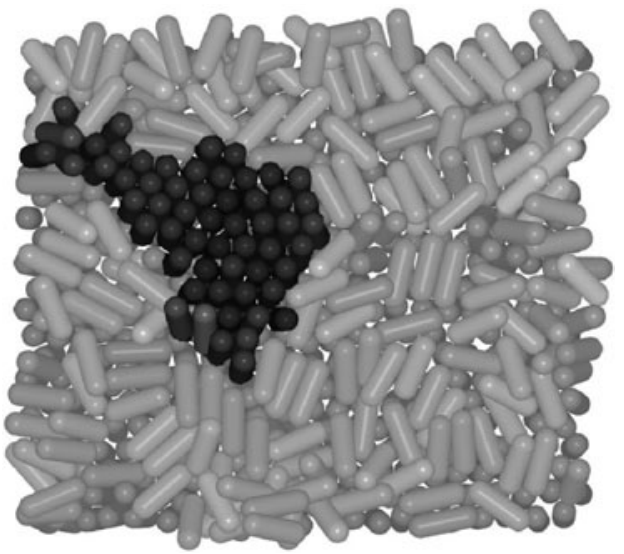

Figure 6. Configuration snapshot of second-layer growth: only those particles are shown that are adjacent to the top surface of a lamellar crystal close to the solid surface (seen from the top). Most particles lie flat (light) but, in addition, a crystalline cluster of about 50 rods has formed (dark).

crystallites. The present simulations suggest that this experimental observation is a logical consequence of the fact that, at sufficiently high supersaturation, multilayer growth is still kinetically inhibited, due to orientational 'self-poisoning' of the top and bottom growth surfaces, whereas lateral growth proceeds relatively unhampered.

However, our results hold only for short rods. The formation of smectic and solid aggregates of longer rods $(L / D \geqslant 3)$ proceeds via the nematic phase and therefore follows a different pathway.

The present simulations suggest that, experimentally, the growth of colloidal crystals of short rod-like particles can be greatly enhanced by applying a field that pre-aligns the rods. In particular, it should facilitate the growth of multilayer crystals under conditions where a single-layer lamellar crystal is thermodynamically unstable.

\section{Acknowledgments}

This work is part of the research programme of 'Stichting Fundamenteel Onderzoek der Materie (FOM)' which is financially supported by 'Nederlandse Organisatie voor Wetenschapelijke Onderzoek (NWO)'. TS acknowledges support through a European Community Marie Curie Fellowship.

\section{References}

[1] Auer S and Frenkel D 2001 Prediction of absolute crystal-nucleation rate in hard-sphere colloids Nature 409 1020

[2] ten Wolde P R, Ruiz-Montero M J and Frenkel D 1996 Simulation of homogeneous crystal nucleation close to coexistence Faraday Discuss. 10493

[3] Dogic Z and Fraden S 2001 Development of model colloidal liquid crystals and the kinetics of the isotropicsmectic transition Phil. Trans. R. Soc. A 359997

[4] Frenkel D and Schilling T 2002 Smectic filaments in colloidal suspensions of rods Phys. Rev. E 66041606

[5] Asthagiri D, Lenhoff A M and Gallagher D T 2000 Role of competitive interactions in growth rate trends of subtilisin s88 crystals J. Cryst. Growth 212543

[6] Ungar G, Mandal P K, Higgs P G, de Silva D S M, Boda E and Chen C M 2000 Dilution wave and negative-order crystallization kinetics of chain molecules Phys. Rev. Lett. 854397 
[7] Bolhuis P and Frenkel D 1997 Tracing the phase boundaries of hard spherocylinders J. Chem. Phys. 106666

[8] Downton M T and Allen M P 2003 Computer simulation of liquid crystal surface modification Preprint available from http://eprints.csc.warwick.ac.uk/

[9] Maeda H and Maeda Y 2003 Liquid crystal formation in suspensions of hard rodlike colloidal particles: direct observation of particle arrangement and self-ordering behaviour Phys. Rev. Lett. 90018303 